\title{
Adult Attachment and the Perception of Emotional Expressions: Probing the Hyperactivating Strategies Underlying Anxious Attachment
}

\author{
R. Chris Froley \\ University of Illinois at Urbana-Champaign \\ Paula M. Niedenthal \\ National Center for Scientific Research and University of \\ Clermont-Ferrand \\ Michael Marks, Claudia Brumbaugh, and \\ Amanda Vicary \\ University of Illinois at Urbana-Champaign
}

\begin{abstract}
According to adult attachment theory, individual differences in attachment-related anxiety reflect variation in individuals' vigilance to cues relevant to appraising and monitoring the availability and responsiveness of significant others. To investigate this assumption, the authors adopted a morph movie paradigm in which participants were shown movies of faces in which an emotional facial expression changed gradually to a neutral one (Study 1) or a neutral expression changed to an emotional one (Studies 2-4). Participants were asked to judge the point at which the emotional expression had disappeared or emerged, respectively. Individuals who were highly anxious with respect to attachment were more likely to perceive the offset (Study 1) as well as the onset (Studies 2 and 3) of the facial expressions of emotion earlier than other people. Moreover, this heightened state of vigilance may have led to poorer

Address correspondence to: R. Chris Fraley, Department of Psychology, 603 E. Daniel St., University of Illinois, Champaign, IL 61820. E-mail: rcfraley@uiuc.edu.

Journal of Personality 74:4, August 2006

(C) 2006, Copyright the Authors

Journal compilation (C) 2006, Blackwell Publishing, Inc.

DOI: $10.1111 / \mathrm{j} .1467-6494.2006 .00406 . x$
\end{abstract}


accuracy in judging facial expressions of emotion (Study 3), an effect that was reversed when anxious individuals were required to watch the movies for the same length of time as less anxious participants (Study 4). The results indicate that variation in attachment anxiety reflects, in part, differences in vigilance to cues of social and emotional significance.

One of the fundamental assumptions of attachment theory is that there are individual differences in the way people organize their thoughts, feelings, and behaviors in close relationships and that those differences are rooted, in part, in their representations of experiences in past intimate relationships (Bowlby, 1969, 1973; Hazan \& Shaver, 1987). During the past two decades, an extraordinary amount of research on personality processes and individual differences has been conducted within this tradition. Researchers have found, for example, that individual differences in attachment security are associated with the way in which people regulate their behavior prior to a naturally occurring separation from their partners (Fraley \& Shaver, 1998), the way they experience and manage conflict in intimate relationships (Simpson, Rholes, \& Phillips, 1996), the strategies they use for mental control (Mikulincer, Dolev, \& Shaver, 2004), and the extent to which they disclose information of personal significance (Mikulincer \& Nachshon, 1991; see J. A. Feeney, 1999, for a more thorough review).

Although the bulk of this research has focused on the way in which people with different attachment styles regulate their emotions and behavior, attachment theory also holds that the cognitive processes underlying these differences can operate preemptively, playing a role in how individuals perceive information that is relevant to attachment concerns (Fraley, Garner, \& Shaver, 2000). In other words, working models are thought to influence not only the way people organize their behavior but also the way in which they perceive, attend to, and process information of emotional significance (see Collins \& Read, 1994; B. C. Feeney \& Cassidy, 2003; Niedenthal, Brauer, Robin, \& Innes-Ker, 2002). The objective of the research reported here is to investigate the relationship between a participant's attachment style and his or her ability to perceive facial expressions of emotion. Specifically, we used a "morph movie" paradigm (see Feldman Barrett \& Niedenthal, 2004; Niedenthal, Halberstadt, Margolin, \& Innes-Ker, 2000; Niedenthal et al., 2002) to 
study sensitivity and vigilance to emotional signals. Participants were shown movies of faces in which the facial expression that was displayed changed gradually from an emotional expression to a neutral one (Study 1) or from a neutral expression to an emotional one (Studies 2-3) and were asked to judge the point at which the emotional expression had changed. This method allowed us to investigate the potential role that individual differences in attachment play in interpersonal attentional and perceptual processes.

We begin by summarizing some of the key ideas in attachment theory, especially as they pertain to the cognitive processes that might influence the perception of social and emotional stimuli. Next, we discuss the morph movie paradigm in more depth and explain why it is a valuable method for the study of attachment, emotion, and perception. We then present four empirical studies designed to probe the relationship between individual differences in perceptual vigilance and adult attachment. It is our hope that these studies will help elucidate some of the cognitive dynamics that underlie anxious attachment, as well as advance theory and research on the interface of perception, emotion, and personality more generally.

\section{The Dynamics of the Attachment System}

One of Bowlby's (1969) many important contributions was his account of the way in which the attachment behavioral system functions. Bridging ideas from the study of control mechanisms and ethology (e.g., Hinde, 1966), Bowlby argued that one of the key control processes in the system is responsible for answering the following question: Is the attachment figure nearby, accessible, and attentive? If the individual perceives the answer to this question to be "yes," he or she feels loved, secure, and confident and, behaviorally, is likely to explore his or her environment freely. If, however, the individual perceives the answer to this question to be "no," he or she experiences anxiety and, behaviorally, is likely to exhibit attachment behaviors ranging from simple visual searching on the low extreme to active following and vocal signaling to the other. These behaviors continue until either the individual is able to reestablish a desirable level of physical or psychological proximity to the attachment figure or until he or she becomes fatigued, as may happen in the context of a prolonged separation or loss. 
A central assumption of this model is that there is a cognitive process that plays a role in appraising and monitoring the environment for cues regarding the availability and accessibility of significant others (see also Bretherton, 1985; Fraley \& Shaver, 2000; Pietromonaco \& Feldman Barrett, 2000). According to contemporary models of individual differences in attachment organization, this appraisal system is more sensitive for some people than others, reflecting, in part, differences in individual interpersonal histories (Fraley \& Shaver, 2000; Fraley \& Spieker, 2003; Mikulincer \& Shaver, 2003; Shaver \& Mikulincer, 2002). Importantly, variation in the sensitivity of this monitoring process, which Mikulincer and Shaver (2003) refer to as hypervigilance, is thought to account for differences in the extent to which individuals worry about whether they are loved by significant others, and, ultimately, whether they are themselves lovable (i.e., variation in attachment-related anxiety).

A large body of research has now documented the implications of individual differences in attachment anxiety for relationship functioning (see Feeney, 1999, for a review). Simpson and colleagues (1996), for example, showed that compared to less anxious women, highly anxious women experienced more anger and hostility toward their partners when discussing an area of conflict in their close relationships. However, there is little research that has addressed the question of whether variation in attachment-related anxiety is rooted in individual differences in vigilance to affective cues. One noteworthy exception is a study reported by Mikulincer, Gillath, and Shaver (2002). These researchers found that highly anxious people were faster to make lexical decisions in response to the names of their attachment figures. This finding suggests that anxious adults may be more sensitive to cues that are relevant to the functioning of the attachment system. One of the goals of the present research was to investigate further the interface of vigilance and attachment by employing a novel paradigm for studying the way in which people perceive affective stimuli. We describe this paradigm in the next section.

\section{MORPH MOVIES AND PERCEPTIONS OF FACIAL EXPRESSION OF EMOTION}

Niedenthal and her colleagues (Niedenthal, Brauer, Halberstadt, \& Innes-Ker, 2001; Niedenthal et al., 2000) have developed a useful 
method for investigating the interface between cognition, perception, and emotion. In the "morph movie" task, participants watch a 100frame computerized movie in which a face initially displaying a specific emotional expression (e.g., happiness) gradually evolves into one displaying a neutral expression. Participants are asked to stop the movie at the frame at which they believe the emotion has dissipated. This offset frame is the data point of interest.

The morph movie technique offers a valuable way to study emotion perception for at least three reasons. First, because the emotional expression disappears gradually over the course of the movie, there comes a point at which one's perception is equally guided by the objective features of the stimulus and the top-down inferential processes of theoretical interest. Second, because the method involves the perception of facial expressions of emotion rather than lexical items (i.e., emotion words) or other kinds of stimuli, it has a high degree of ecological validity. A key task of social life is understanding the motivational and affective states of others, and cues to those states can often be gleaned from facial expressions (Fridlund, 1992). Finally, the response in the task is nonverbal. Rather than attaching verbal labels to percepts, participants indicate the frame in a movie at which they perceive a certain event, such as the onset or offset of a dynamic facial expression (see Niedenthal \& Halberstadt, 2003, for discussion).

In one of the first studies using the morph method, Niedenthal et al. (2000) found that participants in whom happiness had been experimentally induced judged the offset of happy expressions in the morph movies at a later frame than participants in a control condition. Based on these and similar findings (e.g., Niedenthal et al., 2001), Niedenthal and her colleges have concluded that the offset judgment partly reflects the degree of sensitivity with which people are able to process emotional expressions. Specifically, they proposed that people who are experiencing an emotional state are more sensitive to cues congruent with that state and, consequently, see these cues as persisting for a longer period of time in the context of an ambiguous-neutral expression than those experiencing a different state.

In a study more closely related to the present research, Niedenthal and her colleagues (2002) used the morph movie task to investigate the relationship between adult attachment styles and perceptions of facial expressions of emotion. Using a categorical measure of 
attachment styles (i.e., Relationship Questionnaire, RQ; Bartholomew \& Horowitz, 1991), they found that preoccupied adults tended to judge the offset of emotional expressions later than secure adults and that fearful adults perceived the offset significantly earlier than their secure counterparts (Study 1). They interpreted these findings as suggesting that preoccupied individuals are habitually more sensitive to and efficient in their processing of emotional signals and thus detect evidence of a facial expression with less perceptual evidence than others (i.e., compared to others, they see an emotional expression linger for a longer period of time). They also suggested that fearful adults judged the offset of the emotional expressions sooner than others because their fearful avoidance of intimacy is associated with less sensitivity to emotional cues.

\section{Extending the Paradigm}

One of the objectives of the present research was to replicate the Niedenthal et al. (2002) findings, using a dimensional, as opposed to a categorical, measure of individual differences in attachment-related anxiety and avoidance (e.g., Brennan, Clark, \& Shaver, 1998; Fraley \& Waller, 1998). More importantly, however, we also extended the basic paradigm in some significant new directions. First, we sought to clarify the meaning of the offset scores in the morph movie task. Niedenthal and her colleagues have interpreted late offset scores in morph movies as reflecting sensitivity in processing. This suggests that anxious individuals, who are assumed to be highly vigilant to emotional cues, should see an emotional expression persisting into an increasingly neutral expression for a longer period of time than others (see Niedenthal et al., 2002). However, it is also possible that vigilance has its effects in a different way. Namely, highly vigilant people may be more sensitive to emotional change, leading them to judge the offset of emotions earlier than others. This conceptualization would lead to an entirely different interpretation of the findings of Niedenthal and colleagues (2002). Perhaps, in fact, the fearful participants in that study were highly vigilant, but their vigilance was in detecting change rather than continued evidence of an emotional expression.

Unfortunately, the standard morph task does not allow one to distinguish between these alternative interpretations, making it unclear whether early offset judgments reflect a lack of sensitivity to 
evidence of an emotional expression or heightened sensitivity to change in an expression. To help resolve this ambiguity, in the first study reported here we adopted the original emotional expressionto-neutral morph movie method in which participants judge the offset of the initial emotional expression. Then in three additional studies, we reversed the direction in which the morph movies were played (i.e., the movies began with an actor exhibiting a neutral expression that gradually evolved into one of anger, happiness, or sadness) and asked participants to judge the onset of emotion (e.g., Feldman Barrett \& Niedenthal, 2004). If highly anxious adults are truly more sensitive to cues of emotion per se, they should judge the offset of emotional expressions later than others and judge the onset of emotional expressions sooner than others. However, if highly anxious adults are more sensitive at detecting evidence of emotional change, they then should see both the offset and onset of emotional expressions earlier than nonanxious adults.

We also adapted the morph movie task in a second way. In previous research with these kinds of movies, participants were able to view the entire movie without restraint (i.e., they could rewind or fast forward through the movie as they saw fit). As such, participants had full knowledge of the way in which the emotional expression unfolded when they made their judgments. But what would happen if participants were given a speeded decision task in which they were asked to stop the morph movie as soon as they identified a change in the emotional expression, such as a change from a neutral expression to one of anger, happiness, or sadness? This kind of task should provide another means for investigating the role that perceptual processes play in individual differences in attachment. In Study 3, we modified the task in this manner to determine not only how early emotions are perceived by individuals varying in attachment style but also how accurately they are able to judge the emotions portrayed. In Study 4, we programmed the computer to control the duration of the morph movies so that we could separate accuracy from the effects of vigilance.

A final goal of this research was to resolve a previously unacknowledged paradox regarding the Niedenthal et al. (2002) study. Niedenthal et al. (2002) found that fearful and secure people tended to have lower offset scores than preoccupied and dismissing people. This is an unusual finding for two reasons. First, it suggests that highly secure and fearful people may have something in common, an 
observation that is most unusual in adult attachment research (see Shaver \& Mikulincer, 2002, for a review). Second, within contemporary two-dimensional models of individual differences in attachment, both highly preoccupied and fearful people are highly anxious about attachment concerns. Thus, if there is a vigilance mechanism that is common to these attachment styles, preoccupied and fearful individuals should exhibit similar patterns of behavior in the morph task. To model the Niedenthal et al. (2002) data within the twodimensional framework, it would be necessary to have a negatively weighted interaction term between anxiety and avoidance, such that the effect of anxiety on offset scores was negative among highly avoidant people and positive among less avoidant people. Unfortunately, it is difficult to untangle the main and interactive effects of the attachment dimensions with categorical measures alone. As such, in this research we tested our key predictions regarding attachmentrelated anxiety, but we also tested the interaction between anxiety and avoidance to determine whether the original Niedenthal findings would hold.

\section{Avoidant Attachment}

Although the primary goal of this research was to test the theoretical assumption that hypervigilance to affective signals underlies anxious attachment (see Fraley \& Shaver, 2000; Fraley \& Spieker, 2003), this research also provides a means to determine whether vigilance processes underlie attachment-related avoidance. There are at least two competing hypotheses that can be derived from attachment theory regarding the association between avoidance and performance in the morph movie task. One hypothesis is that highly avoidant people are more sensitive to emotional changes because they need to be able to detect interpersonal cues in an efficient manner to defend themselves effectively. If this hypothesis is correct, avoidant individuals should judge the onset and offset of emotions in morph movies in a way that reflects vigilance. An alternative hypothesis is that highly avoidant people defend themselves against attachment-related emotions in part by blunting the perception of interpersonal cues. If this hypothesis is correct, we might expect avoidant individuals to judge the onset and offset of emotions in a way that reflects a lack of sensitivity affective signals. 


\section{OVERVIEW OF THE PRESENT STUDIES}

The primary objective of this research was to examine the relationship between attachment orientation and the perception of facial expressions of emotion. In Study 1, we sought to replicate the basic effects reported by Niedenthal et al. (2002), but to do so using dimensional measures of adult attachment orientation. In Studies 2-4, we extended this paradigm by reversing the order in which the morph movies were played, such that faces initially displayed a neutral expression that gradually changed into an emotional one. This modification allowed us to assess more clearly the role that perceptual vigilance plays in adult attachment. In Studies 3 and 4, we examined the accuracy of participants' perceptions to determine whether heightened vigilance facilitates or inhibits an individual's ability to identify emotional expressions.

\section{STUDY 1}

\section{Method}

\section{Participants}

One-hundred and nineteen people completed the study as part of an online experiment. The data were collected through a Web site designed "to assess your attachment style in different relationships as well as the way in which you perceive emotional cues." The study was hosted on the first author's Web site, www.yourpersonality.net, which contains a variety of Web studies and demonstrations regarding personality, attachment, and close relationships. The site can be found via Web searches for free personality tests and receives approximately 100 visitors a day (although not all visitors participate in each study and/or exercise posted on the Web site). The sample was composed primarily of women $(77 \%)$. The average age of participants was $25(S D=8)$. The majority of participants were from the United States $(n=87)$, with the remainder of the sample participating from Great Britain $(n=8)$, Canada $(n=4)$, or elsewhere. (For an in-depth comparison of Web-based samples and more commonly used undergraduate samples, please see Gosling, Vazire, Srivastava, \& John, 2004.)

\section{Materials and Procedure}

Participants answered 40 questions designed to assess their attachment orientation across four kinds of intimate relationships (i.e., relationships 
with mother, father, romantic partners, and best friends). For each item, participants were asked to click on a continuous scale to indicate the extent to which they agreed or disagreed with the item. The items were derived from the ECR-R, an attachment inventory designed to assess the two fundamental dimensions underlying attachment patterns (Fraley, Waller, \& Brennan, 2000). Because we did not make differential predictions about how attachment as assessed in different relational contexts may be related to the way emotional expressions are processed, we separately averaged responses to the anxiety and avoidance items across the four relational targets to create composite indices of global attachmentrelated anxiety and avoidance. The alphas for both scales were greater than or equal to 0.89 across the four studies.

The morph movies were generated by taking a digital image of an actor instructed to express happiness, sadness, or anger and morphing that image into one in which the same actor was instructed to pose in an emotionally neutral fashion. The images were taken from the MacBrain Face Stimulus Set, an archive of emotion faces that have been pretested for a variety of characteristics. ${ }^{1}$ The emotions conveyed in the specific images we selected had been correctly identified by over $99 \%$ of children and adults who had been asked to judge the emotional expressions in previous work. We used a digital morphing program (Abrosoft, 2004) to produce a digital movie composed of 100 facial composites, such that successive composites changed in a mathematically equal degree toward the neutral face. (See Figure 1 for an example of some of the morph movies.) Three separate faces were used for each of three emotions (i.e., anger, sadness, and happiness), yielding nine morph movies in all. The movies were exported as Flash files (Macromedia, 2000), enabling them to be downloaded relatively rapidly and presented in a manner that is viewable in most Web browsers. The movies were displayed in a $170 \times 225$ pixel frame. The order in which they were shown was randomized for each participant.

The instructions stated that participants would be viewing a series of nine movies in which an actor begins by expressing an emotion (anger, happiness, or sadness) that gradually fades. Participants were instructed to watch the full movie once and then to watch it a second time and press the "stop" button at the point at which they thought that the person in the clip was no longer expressing the original emotion. Once the movie had loaded, participants watched the complete movie. The movie then

1. Development of the MacBrain Face Stimulus Set was overseen by Nim Tottenham and supported by the John D. and Catherine T. MacArthur Foundation Research Network on Early Experience and Brain Development. Please contact Nim Tottenham at tott0006@tc.umn.edu for more information concerning the stimulus set. 

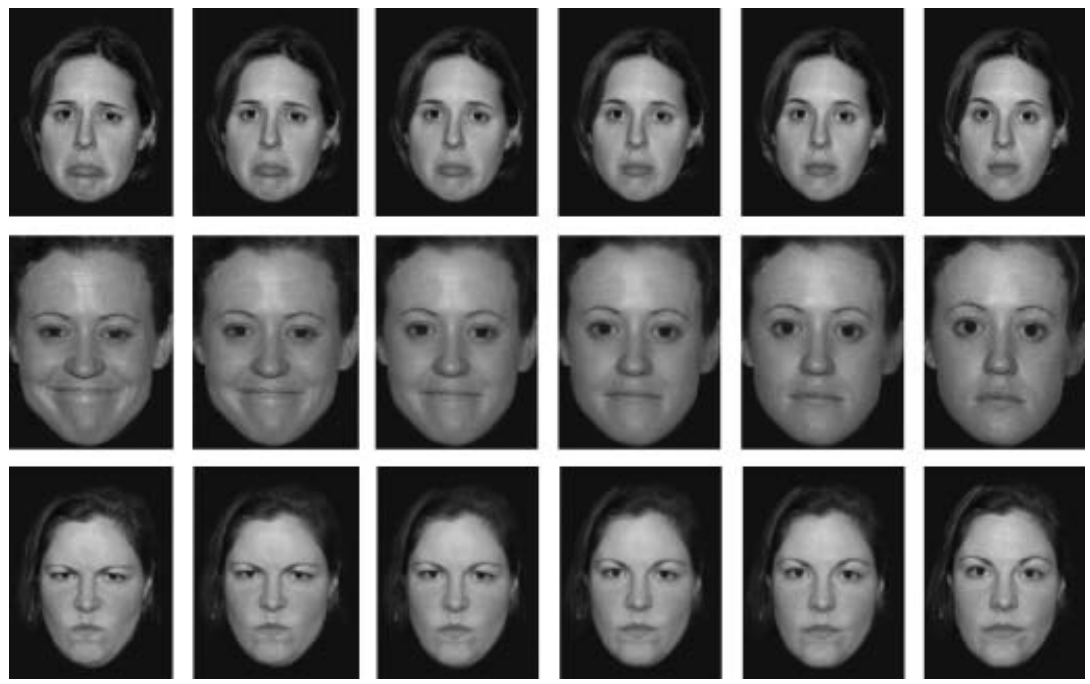

Figure 1

Six approximately equally spaced frames, including first and last, of a sad-to-neutral morph movie (Row 1), a happy-to-neutral morph movie (Row 2), and an angry-to-neutral morph movie (Row 3).

played again, and participants stopped it at the point at which they thought the original expression had disappeared from the face. Participants were allowed to fine-tune their stopping point by pressing "back" and "forward" buttons to move through the movie one frame at a time. When the participant was satisfied with the offset frame, he or she pressed a button to advance to the next movie.

When all nine trials had been completed, the participant was asked to provide some demographic information. After the data had been submitted, the Web script provided the participant with detailed feedback about his or her attachment patterns in different relationships, as well as a summary of the point at which he or she stopped the morph movies and the point at which other participants in the sample had done so.

\section{Results}

Table 1 reports the correlations between attachment-related anxiety and avoidance and the average offset frame for angry, happy, and sad movies. ${ }^{2}$ As can be seen, highly anxious people tended to judge the

2. Participant sex was not related to the morph movie scores in this study or the studies that follow. 


\section{Table 1}

Correlations Between Attachment Style and the Average Offset Scores of Emotions in the Morph Movies in Study 1

\begin{tabular}{lcc}
\hline & \multicolumn{2}{c}{ Attachment } \\
\cline { 2 - 3 } Emotion & Anxiety & Avoidance \\
\hline Anger & $-.23^{*}$ & -.11 \\
Happiness & $-.19^{*}$ & -.12 \\
Sadness & $-.24^{*}$ & -.19 \\
Composite & $-.23^{*}$ & -.15 \\
\hline
\end{tabular}

Note. Higher offset scores indicate that the participant judged the offset of the emotion later, as opposed to earlier, in the movie.

$* p<.05$.

offset of the emotional expressions sooner (i.e., at an earlier frame) than less anxious people. This was the case regardless of what kind of emotion was being considered. As such, we created a composite offset index by averaging the frame offsets across the nine movies (alpha $=.95)$. The correlation between this index and anxiety was -.23 . When we regressed the index onto anxiety and avoidance simultaneously, the regression model was statistically significant, $F(2$, $115)=3.34, p<.05$. The average offset frame across participants (i.e., the constant in the regression model) was 70.9. Attachment-related anxiety was negatively associated with frame offset $(\mathrm{B}=-5.37$, $\beta=-.25, p<.05)$, but avoidance was not associated with frame offset $(\mathrm{B}=.62, \beta=.03, n s)$. Participants who were $1 S D$ above the mean in anxiety had an average offset frame of 65.6, whereas those $1 S D$ below the mean in anxiety had an average onset frame of 76.3 .

We also tested the interaction between anxiety and avoidance to see whether we could replicate the effects reported by Niedenthal et al. (2002). To model their findings, we needed to observe a negatively weighted interaction term between anxiety and avoidance. However, the addition of the interaction term to the model did not lead to a statistically significant increase in the $R^{2}(F[1,114]=.35$, $n s ; \mathrm{B}=1.24, \beta=.06){ }^{3}$

3. We also tested the interaction term in the studies that follow. In no case was the interaction between anxiety and avoidance a significant predictor of the dependent measures. 


\section{STUDY 2}

The results from Study 1 suggest that highly anxious individuals tend to judge the offset of facial displays of emotion earlier than less anxious people. Within the interpretive framework outlined by Niedenthal et al. (2001), this finding suggests that highly anxious people are less efficient in processing emotional cues than others - a finding that might seem a bit peculiar within the context of attachment theory. Another possible interpretation of this finding, however, is that anxious people are hypervigilant to social and emotional cues that signal change in emotional states. In other words, judging the offset of the emotion early in the movie may not necessarily reflect a tendency to be less sensitive to persisting signals of emotionit may reflect a heightened sensitivity to changes in emotional cues.

How can these alternative interpretations be tested? One way to do it is to reverse the direction in which the movies are played, such that they begin with a face displaying a neutral expression that gradually evolves into one of anger, happiness, or sadness and then ask participants to judge the onset of the emotion (see Feldman Barrett $\&$ Niedenthal, 2004). If anxious people are sensitive to emotional change, they should judge the onset of emotions prior to others. Study 2 was designed to test this hypothesis.

\section{Method}

\section{Participants}

Data were collected via the Internet through the same means described in Study 1. Participants were 127 people who had not previously participated in the research project. The sample was primarily composed of women $(76 \%)$. The average age of participants was $24(S D=8)$. The majority of participants were from the United States $(n=101)$, with the remainder of the sample participating from Canada $(n=11)$, Great Britain $(n=5)$, or elsewhere.

\section{Materials and Procedure}

The methods were identical to those for Study 1, with one critical exception: the morph movies were reversed so that each movie began with a face displaying a neutral expression that gradually evolved into one indicative of anger, sadness, or happiness. After watching the movie all the way through, participants were instructed to stop it at the point at which they believed the actor was experiencing an emotion. As before, 
participants were allowed to fine-tune their stopping point by advancing the movie frames forward or backward before moving on to the next movie.

\section{Results}

Table 2 reports the correlations between attachment-related anxiety and avoidance and the average onset frame for angry, happy, and sad movies. As can be seen, highly anxious people tended to judge the onset of the emotional expressions much sooner (i.e., at an earlier frame) than those who were less anxious. This finding is consistent with the hypothesis that the morph movie task captures sensitivity to changes in emotion and that highly anxious people are more vigilant to these cues. As before, the effect was in the same direction regardless of what kind of emotion was being judged. The correlation between the composite onset index (alpha $=.96)$ and anxiety was -.20 $(p<.05)$. When we regressed this index onto anxiety and avoidance simultaneously, the regression model was statistically significant, $F(2,115)=3.34, p<.05$. The constant in this equation was 49.2 , indicating that participants, on average, tended to judge the onset of the emotion about half-way though the morph movie. Attachmentrelated anxiety was negatively associated with frame offset $(\mathrm{B}=-4.92, \beta=-.25, p<.05)$; avoidance was not associated with frame offset $(\mathrm{B}=.55, \beta=.03, n s)$. Participants who were $1 S D$ above the mean in anxiety had an average onset frame of 44.3 , whereas

Table 2

Correlations Between Attachment Style and the Average Onset Scores for the Perception of Emotion in the Morph Movies in Study 2

\begin{tabular}{lcc}
\hline & \multicolumn{2}{c}{ Attachment } \\
\cline { 2 - 3 } Emotion & Anxiety & Avoidance \\
\hline Anger & $-.22^{*}$ & -.16 \\
Happiness & -.13 & -.07 \\
Sadness & $-.21^{*}$ & -.16 \\
Composite & $-.20^{*}$ & -.16 \\
\hline
\end{tabular}

Note. Higher onset scores indicate that the participant judged the onset of the emotion later, as opposed to earlier, in the movie.

$* p<.05$. 
those $1 S D$ below the mean in anxiety had an average onset frame of 54.1 .

\section{STUDY 3}

Thus far, the results suggest that highly anxious individuals are more sensitive to changes in emotional-expressive cues than less anxious adults. Anxious adults judged both the offset (Study 1) and the onset (Study 2) of emotional expressions as occurring sooner than did others. The finding that highly anxious people are more vigilant to changes in emotional expression suggests that they may be more accurate in judging the emotional states of others. Empirical research on attachment and interpersonal accuracy, however, is somewhat mixed. Tucker and Anders (1999), for example, found that anxiously attached men were less accurate than nonanxious men in assessing their partners' feelings about their relationship. Simpson, Ickes, and Grich (1999), in contrast, found that highly anxious adults, relative to less anxious adults, were quite adept at gauging the thoughts and feelings of their partners in an empathic accuracy task.

The purpose of Study 3 was to determine whether the heightened vigilance of anxious adults allows them to judge other peoples' emotional states more accurately. The methods were identical to those of Study 2-with one exception. Participants were not allowed to watch the movie all the way through before deciding on an onset frame for the emotion in question. Instead, participants were asked to judge the onset of the emotion as quickly as possible. Thus, the instructional set forced a trade-off between speed and accuracy. Once the onset judgment had been made, participants stopped the movie and were asked to judge which emotion the actor was experiencing: anger, happiness, or sadness. This method allowed us to replicate conceptually the findings from Study 2 in addition to assessing the accuracy of participants' perceptions of emotional expressions.

\section{Participants}

Data were collected via the Internet through the same means described in Study 1. One hundred and forty-one people who had not participated in the research project previously took part in the study. The sample was primarily composed of women $(80 \%)$. The average 
age of participants was $25(S D=9)$. The majority of participants were from the U.S. $(n=107)$, with the remainder of the sample participating from Canada $(n=12)$, Great Britain $(n=6)$, or elsewhere.

\section{Materials and Procedure}

The methods were the same as those described in Study 2, with the exception that participants were not allowed to view the morph movies all the way through before making their onset judgments. Instead, they were instructed to stop the movie as soon as they perceived the actor to be experiencing one of three emotions: anger, sadness, or happiness. Once the movie was stopped, participants were asked to judge which of the three emotions the actor was experiencing. Participants were not allowed to replay the movie or advance forward to subsequent frames.

\section{Results}

Table 3 reports the correlations between attachment-related anxiety and avoidance and the average onset frame for angry, happy, and sad movies. As we observed previously, highly anxious individuals tended to judge the onset of the emotional expressions much sooner (i.e., at an earlier frame) than those who were less anxious. More-

\section{Table 3}

Correlations Between Attachment Style and the Average Onset Scores for the Perception of Emotion in the Morph Movies in Study 3

\begin{tabular}{lcc}
\hline & \multicolumn{2}{c}{ Attachment } \\
\cline { 2 - 3 } Emotion & Anxiety & Avoidance \\
\hline Anger & -.14 & -.05 \\
Happiness & -.08 & -.01 \\
Sadness & -.13 & -.02 \\
Composite & -.13 & -.03 \\
Hits & $-.20^{*}$ & -.12 \\
\hline
\end{tabular}

Note. Higher onset scores indicate that the participant judged the onset of the emotion later, as opposed to earlier, in the movie. Hits refers to the number of correct emotion judgments.

$* p<.05$. 
over, this pattern emerged regardless of what kind of emotion was being judged; the correlation between the composite onset score (alpha $=.95$ ) and anxiety was -13 . These correlations, although not statistically significant at the .05 level, were in the same direction as those reported previously. When we regressed the composite onset index onto anxiety and avoidance simultaneously, the regression model was not statistically significant, $F(2,142)=1.47, p=.23$. The constant in this equation was 38.89 , indicating that participants, on average, were judging the onset of emotional expressions more quickly in this speeded task compared to Study 2. Attachmentrelated anxiety was negatively, but not significantly, associated with frame offset $(\mathrm{B}=-3.95, \beta=-.18, p<.10)$; avoidance was not associated with frame offset $(\mathrm{B}=1.80, \beta=.08, n s)$.

We quantified accuracy as the total number of movies that were judged correctly. ${ }^{4}$ (The frequency of hits was highly correlated across the three kinds of emotion movies, so, in the analyses that follow, we focus on the accuracy across all the movies.) Importantly, highly anxious people tended to have a lower degree of accuracy than less anxious people $(r=-.20, p<.05)$. When we regressed this index onto anxiety and avoidance simultaneously, the regression model was significant $F(2,142)=2.8, p<.05$. The constant in this equation was 6.7 , indicating that participants, on average, were able to judge the expressions fairly accurately, getting approximately 7 out of 9 correct. Attachment-related anxiety was negatively associated with accuracy $(\mathrm{B}=-.46, \beta=-.20, p<.05)$. Individuals $1 S D$ above the mean in anxiety tended to be 1 face less accurate (6.23) than those 1 $S D$ below the mean in anxiety (7.15). Avoidance was not related to accuracy $(\mathrm{B}=.02, \beta=.01)$.

\section{STUDY 4}

The results of Study 3 suggest that although highly anxious people are sensitive to changes in others' emotional states, this sensitivity also leads them to jump to emotional conclusions, so to speak, ultimately undermining their ability to perceive emotions accurately.

4. It should be noted that highly anxious people were not any more likely than less anxious people to rely upon negative responses (i.e., anger, sadness) in guessing the emotions. Thus, the finding of poorer accuracy is not a result of anxious people adopting a different response bias than others. 
These findings raise the question of whether the decreased accuracy among highly anxious adults reflects the fact that they make their judgments more quickly than other people (i.e., with less information) or whether they are truly less able to evaluate others' emotional states. In Study 4 we addressed this question by holding constant the amount of information that people received by having the computer, rather than the participant, control the point at which each movie stopped. Thus, some movies only played for a few frames, thereby providing the participant with little objective information with which to judge the emotional state of the actor, whereas other movies played for a longer period of time, thereby providing the participant with more objective information.

If it is the case that the poorer accuracy of highly anxious individuals is due to their tendency to make judgments without sufficient information, we should find that when they receive the same amount of information as everyone else, their accuracy does not suffer. Of course, there are other possibilities as well. It may be the case that the vigilance of anxious adults actually allows them to make more accurate judgments as long as they are given the same information as everyone else. Another alternative is that anxious adults' ability to infer the emotional states of others is compromised even when they have the same amount of information as others. In other words, it may be the case that their vigilance not only leads them to make judgments prematurely but also undermines their ability to correctly infer the emotional states of others.

\section{Methods}

\section{Participants}

Data were collected via the Internet using the same basic methods described in Study 1. Participants were 142 people who had not taken part in the research project previously. The sample was primarily composed of women $(76 \%)$. The average age of participants was $28(S D=11)$. The majority of participants were from the United States $(n=94)$, with the remainder of the sample participating from Canada $(n=19)$, Great Britain $(n=13)$, or elsewhere.

\section{Materials and Procedure}

In this study, participants viewed the same nine movies that we had used in the previous studies, but we randomly assigned each movie to have a 
different ending frame. Thus, each participant saw some movies that ended after very few frames (e.g., after 2 frames) and some that were more complete (e.g., ending after 100 frames). The ending frame for each movie was randomly selected, without replacement, from a predetermined distribution of frames: 2, 4, 7, 15, 26, 40, 57, 77, 100. The distribution of end frames was designed to follow approximately a logistic function to ensure that there were more movies played for a shorter period of time than movies played for a longer period of time. We chose to distribute the end frames in this manner because there is less ambiguity in movies that play longer (i.e., the probability of accurately judging an emotion from a movie that plays all the way through is greater than 99\%). Because the "inferential action" lies in cases in which ambiguity exists, we sampled a greater number of end frames from the low end of the spectrum than the high end.

By having each participant view movies that varied in their end frames, we were able to study - separately for each individual - how accuracy varied as a function of the amount of information received. As might be surmised from the prior discussion, the probability of correctly judging an emotion does not vary as a simple linear function of the duration of a morph movie. If a neutral face is gradually morphing into a happy one, for example, it should be fairly clear once the smile becomes evident that the emotion in question is happiness and not sadness or anger. Theoretically, the probability of correctly judging the emotion as the movie plays increases monotonically in a form that can be modeled by a basic logistic function, such as $P=1 /[1+\exp (-A(B-F))]$. In this equation, $P$ represents the probability of perceiving the emotion accurately, which can range from 0 to $1 ; A$ is a rate parameter determining the steepness of the function; $F$ is the frame of the movie, ranging from 1 to 100 ; and $B$ is the threshold parameter - the frame at which a person has a 50-50 chance of correctly judging the emotion portrayed in a morph movie. Some hypothetical "accuracy functions" produced by varying the value of $B$ in this equation are shown in the leftmost panel of Figure 2. ${ }^{5}$ People who are able accurately to infer the emotional state of the actor with a minimal amount of information will have accuracy functions with smaller $B$ values. People who require more information in order to make accurate decisions will have larger $B$ values.

5. In these illustrations and in the empirical estimation routines, we held the parameter $A$ constant at 0.1 because (a) there was little variation in rates per se and (b) the parameter of key interest is the inflection point, $B$, which represents how much information a person needs in order to have a 50-50 chance of judging the emotion accurately. 

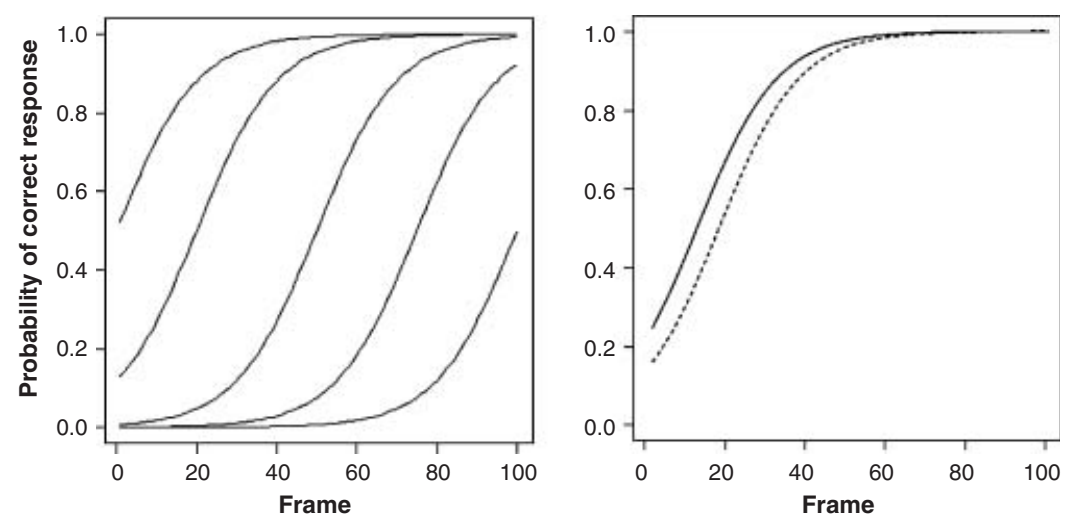

Figure 2

The probability of correctly perceiving an emotional expression as a function of the frame of the morph movie. The leftmost panel illustrates some accuracy functions for hypothetical people with different $B$ values. The values of $B$ for each curve increase from the leftmost to the rightmost curve. The rightmost panel shows the estimated accuracy functions for people $1 S D$ below (dashed curve) and $1 S D$ above (solid line) the mean on attachment-related anxiety.

We estimated the $B$ parameter separately for each participant using a least-squares, grid search estimation routine in S-Plus (MathSoft, 1999). If highly anxious people are more accurate when the amount of information is held constant, their accuracy functions should have lower $B$ values than those for less anxious people. In contrast, if their accuracy is impaired, even when they experience the same amount of information as everyone else, their accuracy functions should have higher $B$ values relative to those for less anxious participants.

\section{Results}

Highly anxious people tended to have slightly lower $B$ estimates $(r=-.18, p<.05)$, indicating that they were more accurate than others in their emotional judgments. The correlation between avoidance and $B$ estimates was -.08 , $n s$. To illustrate the relationship between anxiety and accuracy, the predicted accuracy functions for people $1 S D$ below and above the mean on attachment-related anxiety are illustrated in the rightmost panel of Figure 2. Notice that for a constant amount of information, anxious individuals tend to be more accurate in their judgments than less 
anxious individuals. The differences between them, however, diminish as the amount of information increases. These results suggest that when anxious adults are given the same amount of information as other people, they are able to judge the emotional states of others more accurately.

\section{GENERAL DISCUSSION}

One of the themes of contemporary research on adult attachment concerns the psychodynamics of personality processes - the way in which perception, emotion, and cognition interact with one another to serve motivational goals (Shaver \& Mikulincer, 2002). The objective of the present research was to contribute to this line of research by investigating the role of perceptual vigilance in individual differences in attachment-related anxiety. Using the morph movie paradigm, we found that highly anxious adults were more likely to perceive the offset of facial expressions of emotion earlier than less anxious adults (Study 1). In Study 2, we reversed the morph movie task such that participants were instructed to judge the onset of emotions. We found that highly anxious adults perceived the onset of emotions earlier than less anxious adults. In Study 3, participants made their onset judgments as quickly as possible and, once the judgment had been made, indicated which emotion was being expressed. The pattern of results indicated that anxious adults were impaired in their ability to perceive the facial expressions accurately. In other words, they made more perceptual errors than less anxious adults. In Study 4, we varied within participants the amount of information received (i.e., by presenting them with either few or many frames of a given morph movie) and assessed their ability to perceive the displayed expression accurately. Under these conditions, highly anxious people performed slightly better than less anxious people.

Taken together, these findings suggest that individual differences in vigilance to changes in emotional expression partly underlie variation in attachment-related anxiety. Moreover, this heightened vigilance appears to have ironic effects. Although it allows anxious people to be sensitive to emotional changes, it also leads them to make more errors than other people. This "hair trigger" style of perceptual sensitivity may be one reason why highly anxious people 
experience greater conflict in their interpersonal relationships. Researchers have found that anxious adults tend to have more frequent arguments with their partners (Levy \& Davis, 1988), experience more negative affect in their relationships (Simpson et al., 1996), and are more susceptible to relationship dissolution (Kirkpatrick \& Hazan, 1994). If anxious individuals are more sensitive to interpersonal cues that indicate changes in other peoples' emotional states, they may respond to those changes before having the opportunity to evaluate the situation properly. By jumping to emotional conclusions, they may make more errors in judgment and perpetuate a cycle of conflict and misunderstanding in their relationships. We consider this effect to be "ironic" because when anxious adults are privy to the same amount of information as less anxious people, they appear to be more accurate than less anxious people in their perceptions. This suggests that their hypervigilance could be socially advantageous if harnessed or regulated in an adaptive fashion.

\section{Implications for Models of Attachment Dynamics}

Attachment theory assumes that a critical component of the attachment system is a set of cognitive processes responsible for monitoring the availability and responsiveness of others. More importantly, it is assumed that variation in the functioning of these processes is one reason why some individuals are more anxious with respect to attachment concerns than others. The research presented here provides a novel test of these assumptions. Our data are consistent with the notion that variation in perceptual vigilance underlies attachment-related anxiety. It is noteworthy that our findings were generally consistent regardless of the valence of the emotions being perceived. In other words, highly anxious people were just as sensitive to changes in positive emotions, such as happiness, as they were to negative emotions, such as anger and sadness. This is an important finding because it suggests that, in appraising the availability and responsiveness of others, the attachment system does not differentially weigh signals that are indicative of rejection versus acceptance (see also Zhang \& Hazan, 2002).

An interesting direction for future research is to study the kinds of interpersonal experiences that affect the sensitivity of this monitoring process. Bowlby (1969) suggested that experiences of rejection or 
inconsistent support may lead some people to become more vigilant to others' emotional signals. It should be possible to explore these issues in an experimental fashion by manipulating the supportiveness of individuals' environments (e.g., Collins \& Feeney, 2004) and examining the effect and duration of such manipulations on onset and offset judgments in the morph movie task.

\section{Avoidant Attachment}

Individual differences in attachment-related avoidance were uncorrelated with any of the dependent variables assessed in these studies. At first glance, this may seem peculiar because previous research has found avoidant attachment to be correlated with a variety of affective and interpersonal processes. It is important to note, however, that the morph movie task may not be well suited to tapping the psychological processes underlying avoidant attachment. The morph movie task is thought to tap basic perceptual processes, such as vigilance to interpersonal cues. According to contemporary models of individual differences in attachment (see Fraley \& Shaver, 2000; Fraley \& Spieker, 2003), individual differences in attachment-related anxiety, not avoidance, are thought to be reflections of this kind of vigilance. The present data support this hypothesis, thereby providing evidence for the specificity of the attachment dimensions. Other research on attachment helps to demonstrate the role of avoidance in social and affective interactions. Simpson, Rholes, Oriña, and Gritch (2002), for example, found that highly avoidant people were less responsive to their partner's needs, suggesting that even if highly avoidant people can perceive and recognize basic affective signals just as well as everyone else, they do not necessarily act on that knowledge in an effective manner.

\section{Implications for Research on Perception and Affect}

One of the arguments we developed in the Introduction is that performance on the standard emotion-to-neutral morph movie task is open to several possible interpretations. A late offset score could reflect sensitivity to the presence of emotional cues, but it might also reflect a lack of sensitivity to changes in emotional cues. Because of this ambiguity, it is unclear whether previous findings, such as the relationship between preoccupied attachment and later offset 
judgments (Niedenthal et al., 2002), reflect heightened or diminished attention to emotional cues.

In the present research, we resolved this ambiguity by using both the offset and the onset versions of the morph movie task. We reasoned that if the morph movie task captures variation in peoples' sensitivity to emotional change, then highly vigilant individuals should judge both the offset and the onset of emotional expressions earlier than other individuals. The fact that highly anxious adults did indeed have early offset (Study 1) and onset (Study 2) scores suggests that the original version of the morph movie task captures sensitivity to changes in emotional expressions rather than sensitivity to enduring cues of emotional expressions. This explanation suggests a different interpretation of previous research findings. For example, it suggests that fearful adults (i.e., those who are highly anxious; see Brennan et al., 1998) who had lower offset scores (i.e., they judged the emotion as ending sooner than other people did) in Niedenthal et al.'s (2002) study were more sensitive, rather than less sensitive, to emotional change. It also suggests that dismissing adults (i.e., people who are not anxious, presumably for defensive reasons; see Fraley, Davis, \& Shaver, 1998) were less sensitive rather than more sensitive to emotional change.

\section{Limitations}

Although the methods employed in the present set of studies have some advantages, there are also limitations to the current work. For one, these methods do not allow us to identify how early in the perceptual process attachment-relevant individual differences emerge. It is arguably the case that the perception of facial displays of emotion requires much more complex machinery than the perception of other kinds of stimuli, despite the fact that evidence suggests that the brain may have specialized regions for processing information about faces (Young et al., 1993) and emotion (LeDoux, 1992). Nonetheless, it would seem that social-emotional experiences would be most likely to impact the organization of higher-order cognitive processes rather than lower-order processes or those that are more specialized in function. This point, of course, is debatable, but it is probably the case that the associations we have observed reflect higher-order perceptual and cognitive processes. 
Another limitation of the current research is that we focused on faces of strangers rather than faces of people who play a significant role in our participants' lives. It would be valuable to determine whether these kinds of effects generalize across facial targets that vary in familiarity to the perceiver. Finally, although we have interpreted these data as bearing on the operation of the attachment system per se, it is possible that highly anxious people are vigilant to changes in any kind of stimuli, whether those stimuli are socially relevant or not. Without a condition that focuses on perceptions of nonsocial stimuli (e.g., the color blue morphing into red), this interpretation cannot be ruled out definitely.

In closing, attachment theory has become an important framework for understanding personality processes and individual differences. The present research suggests that some of those differences, namely those concerning anxiety about abandonment and rejection, may be rooted in variation in individual vigilance to social and emotional stimuli.

\section{REFERENCES}

Abrosoft (2004). FantaMorph (Version 2.5) [computer software]. http://www. abrosoft.com/.

Bartholomew, K., \& Howrowitz, L. (1991). Attachment styles among young adults: A test of the four-category model. Journal of Personality and Social Psychology, 61, 226-245.

Bowlby, J. (1969). Attachment and loss: Vol. 1. Attachment. New York: Basic Books.

Bowlby, J. (1973). Attachment and loss: Vol. 2. Separation. New York: Basic Books.

Brennan, K. A., Clark, C. L., \& Shaver, P. R. (1998). Self-report measurement of adult romantic attachment: An integrative overview. In J. A. Simpson \& W. S. Rholes (Eds.), Attachment theory and close relationships (pp. 46-76). New York: Guilford Press.

Bretherton, I. (1985). Attachment theory: Retrospect and prospect. In I. Bretherton \& E. Waters (Eds.), Growing points in attachment theory and research. Monographs of the Society for Research in Child Development (Vol. 50, Serial No. 109, 3-35).

Collins, N. L., \& Feeney, B. C. (2004). Working models of attachment shape perceptions of social support: Evidence from experimental and observational studies. Journal of Personality and Social Psychology, 87, 363-383.

Collins, N., \& Read, S. (1994). Cognitive representations of attachment: The structure and function of working models. In K. Bartholomew \& D. Perlman (Eds.), Attachment processes in adulthood: Advances in personal relationships (Vol. 5, pp. 53-90). London: Kingsley. 
Feeney, B. C., \& Cassidy, J. (2003). Reconstructive memory related to adolescentparent conflict interactions: The influence of attachment-related representations on immediate perceptions and changes in perceptions over time. Journal of Personality and Social Psychology, 85, 945-955.

Feeney, J. A. (1999). Adult romantic attachment and couple relationships. In J. Cassidy \& P. R. Shaver (Eds.), Handbook of attachment: Theory, research and clinical applications (pp. 355-377). New York: Guilford.

Feldman Barrett, L., \& Niedenthal, P. M. (2004). Valence focus and perceptions of facial affect. Emotion, 4, 266-274.

Fraley, R. C., Davis, K. E., \& Shaver, P. R. (1998). Dismissing-avoidance and the defensive organization of emotion, cognition, and behavior. In J. A. Simpson \& W. S. Rholes (Eds.), Attachment theory and close relationships (pp. 249-279). New York: Guilford Press.

Fraley, R. C., Garner, J. P., \& Shaver, P. R. (2000). Adult attachment and the defensive regulation of attention and memory: The role of preemptive and postemptive processes. Journal of Personality and Social Psychology, 79, 816826.

Fraley, R. C., \& Shaver, P. R. (1998). Airport separations: A naturalistic study of adult attachment dynamics in separating couples. Journal of Personality and Social Psychology, 75, 1198-1212.

Fraley, R. C., \& Shaver, P. R. (2000). Adult romantic attachment: Theoretical developments, emerging controversies, and unanswered questions. Review of General Psychology, 4, 132-154.

Fraley, R. C., \& Spieker, S. J. (2003). What are the differences between dimensional and categorical models of individual differences in attachment? Reply to Cassidy (2003), Cummings (2003), Sroufe (2003), and Waters and Beauchaine (2003). Developmental Psychology, 39, 423-429.

Fraley, R. C., \& Waller, N. G. (1998). Adult attachment patterns: A test of the typological model. In J. A. Simpson \& W. S. Rholes (Eds.), Attachment theory and close relationships (pp. 77-114). New York: Guilford Press.

Fraley, R. C., Waller, N. G., \& Brennan, K. A. (2000). An item response theory analysis of self-report measures of adult attachment. Journal of Personality and Social Psychology, 78, 350-365.

Fridlund, A. J. (1992). The behavioral ecology and sociality of human faces. In M. S. Clark (Ed.), Review of Personality and Social Psychology: Vol. 13. Emotion (pp. 90-121). Thousand Oaks, CA, US: Sage.

Gosling, S. D., Vazire, S., Srivastava, S., \& John, O. P. (2004). Should we trust Web-based studies? A comparative analysis of six preconceptions about Internet questionnaires. American Psychologist, 59, 93-104.

Hazan, C., \& Shaver, P. R. (1987). Romantic love conceptualized as an attachment process. Journal of Personality and Social Psychology, 52, 511-524.

Hinde, R A. (1966). Animal behaviour. New York: McGraw-Hill.

Kirkpatrick, L. A., \& Hazan, C. (1994). Attachment styles and close relationships: A four-year prospective study. Personal Relationships, 1, 123-142.

LeDoux, J. E. (1992). Brain mechanisms of emotion and emotional learning. Current Opinions in Neurobiology, 2, 191-198. 
Levy, M. B., \& Davis, K. E. (1988). Lovestyles and attachment styles compared: Their relations to each other and to various relationship characteristics. Journal of Social and Personal Relationships, 5, 439-471.

Macromedia (2000). Macromedia Flash $M X$ [computer software]. San Francisco.

Mathsoft (1999). S-Plus 2000 [computer software]. Data Analysis Products Division, Mathsoft. Seattle, WA.

Mikulincer, M., Dolev, T., \& Shaver, P. R. (2004). Attachment-related strategies during thought suppression: Ironic rebounds and vulnerable self-representations. Journal of Personality and Social Psychology, 87, 940-956.

Mikulincer, M., Gillath, O., \& Shaver, P. R. (2002). Activation of the attachment system in adulthood: Threat-related primes increase the accessibility of mental representations of attachment figures. Journal of Personality and Social Psychology, 83, 881-895.

Mikulincer, M., \& Nachshon, O. (1991). Attachment style and patterns of selfdisclosure. Journal of Personality and Social Psychology, 61, 321-331.

Mikulincer, M., \& Shaver, P. R. (2003). The attachment behavioral system in adulthood: Activation, psychodynamics, and interpersonal processes. In M. P. Zanna (Ed.), Advances in experimental social psychology (Vol. 35, pp. 53-152). New York: Academic Press.

Niedenthal, P. M., Brauer, M., Robin, L., \& Innes-Ker, Å. H. (2002). Adult attachment and the perception of facial expression of emotion. Journal of Personality and Social Psychology, 82, 419-433.

Niedenthal, P. M., Brauer, M., Halberstadt, J. B., \& Innes-Ker, Å. H. (2001). When did her smile drop? Facial mimicry and the influences of emotional state on the detection of change in emotional expression. Cognition and Emotion, 15, 853-864.

Niedenthal, P. M., \& Halberstadt, J. B. (2003). Top-down influences in social perception. In W. Stroebe \& M. Hewstone (Eds.), The European Review of Social Psychology (Vol. 14, 49-76). London: J. Wiley \& Sons.

Niedenthal, P. M., Halberstadt, J. B., Margolin, J., \& Innes-Ker, Å. H. (2000). Emotional state and the detection of change in facial expression of emotion. European Journal of Social Psychology, 30, 211-222.

Pietromonaco, P. R., \& Feldman Barrett, L. (2000). Attachment theory as an organizing framework: A view from different levels of analysis. Review of General Psychology, 4, 107-110.

Shaver, P. R., \& Mikulincer, M. (2002). Attachment-related psychodynamics. Attachment and Human Development, 4, 133-161.

Simpson, J. A., Ickes, W., \& Grich, J. (1999). When accuracy hurts: Reactions of anxiously-attached dating partners to a relationship-threatening situation. Journal of Personality and Social Psychology, 76, 754-769.

Simpson, J. S., Rholes, W. S., Oriña, M. M., \& Grich, J. (2002). Working models of attachment, support giving, and support seeking in a stressful situation. Personality and Social Psychology Bulletin, 28, 598-608.

Simpson, J. A., Rholes, W. S., \& Phillips, D. (1996). Conflict in close relationships: An attachment perspective. Journal of Personality and Social Psychology, 71, 899-914. 
Tucker, J. S., \& Anders, S. L. (1999). Attachment style, interpersonal perception accuracy, and relationship satisfaction in dating couples. Personality and Social Psychology Bulletin, 25, 403-412.

Young, A. W., Newcombe, F., de Haan, E. H. F., Small, M., \& Hay, D. C. (1993). Face perception after brain injury. Brain, 116, 941-959.

Zhang, F., \& Hazan, C. (2002). Working models of attachment and person perception processes. Personal Relationships, 9, 227-237. 
This document is a scanned copy of a printed document. No warranty is given about the accuracy of the copy. Users should refer to the original published version of the material. 